\title{
Turismo como alternativa de desarrollo del distrito de Santa Cruz de Flores, Cañete - Lima
}

\section{TOURISM AS A DEVELOPMENT ALTERNATIVE FOR THE DISTRICT OF SANTA CRUZ DE FLORES, CAÑETE - LIMA}

\author{
MANUEL GODOFREDO ARIAS ESPICHÁN§ \\ Recibido: 16 julio de 2019 / Aceptado: 26 julio de 2019 \\ §acultad de Ingeniería Geológica, Minera, Metalúrgica y Geográfica. Universidad Nacional \\ Mayor de San Marcos (UNMSM)
}

\section{Resumen}

El presente estudio trata de resolver el problema del Distrito de Santa Cruz de Flores, ubicado en la provincia de Cañete, departamento de Lima. Establece que el turismo es una alternativa para el desarrollo económico, puesto que el distrito no reúne todas las condiciones de infraestructura para atender eficientemente al turista. Se recopiló información sobre el tema a desarrollar, seleccionar y depurar información de trabajos desarrollados en el distrito, elaboración de fichas de encuestas. Se realizaron encuestas, entrevistas a los dueños de los restaurantes, hospedajes y hoteles, así como a las empresas de transportes de Lima a Mala y las empresas de transporte local. Encuestas a la población. Se procesó la información en cuadros y tablas, para interpretar, análizar, discutir los resultados, y como parte del estudio se elaboración de planos. La conclusión del trabajo de investigación determinó que la capacidad de carga actual no satisface al turista, porque hay un déficit para el hospedaje del turista en 386 camas en el distrito para un total de 800 turistas que van al distrito. Falta la red de alcantarillado en Azpitia, planos de rutas de ciclismo de aventura y caminata para aprovechar los recursos naturales.

Palabras claves: Puesta en valor, medio de transporte, guía de turismo, demanda turística, desarrollo sustentable

\begin{abstract}
The present study tries to solve the problem of the District of Santa Cruz de Flores, located in the province of Cañete, department of Lima. It states that tourism is an alternative for economic development, since the district does not meet all the infrastructure conditions to efficiently serve tourists. Information will be collected on the subject to be developed, selected and debug information of works developed in the district, preparation of survey sheets. Surveys were conducted, interviews with owners of restaurants, lodgings and hotels, as well as transport companies from Lima to Mala and local transport companies. Population surveys. The information was processed in tables and tables, to interpret, analyze, discuss the results, and as part of the study, plans were drawn up. The conclusion of the research work determined that the current carrying capacity does not satisfy the tourist, because there is a deficit for tourist accommodation in 386 beds in the district for a total of 800 tourists going to the district. Missing sewerage network in Azpitia, plans for adventure cycling routes and hiking to take advantage of natural resources.
\end{abstract}

Keywords: Value enhancement, means of transport, tourism guide, tourism demand, sustainable development

*Correspondencia de autor: Avenida Venezuela s/n Cuadra 34 Ciudad Universitaria. correo 


\section{INTRODUCCIÓN}

Es importante el paisaje natural observar desde lo alto del cerro patrón para ver las playas de León Dormido, Las Totoritas y hasta el balneario de Asia, además poder apreciar el valle del río Mala y los diversos cultivos del valle. La importancia de las rutas de ciclismo de aventura existente y las rutas para caminata de campo en el área rural. También es importante las ruinas pre incas de la Ollería, su danza como las pallas (Chunchos).

Sus famosos vinos y pisco ganadores de premios internacionales, así mismo su gastronomía, como el tamal Florino, el picante Florino, la sopa bruta y carapulcra, yuca a la olla, entre otros potajes.

\section{MATERIALES Y MÉTODOS}

\section{LUGAR DE ESTUDIO}

El Distrito de Santa Cruz de Flores está ubicado en la provincia de Cañete, altura del Km. 82, Antigua Panamericana Sur. Las Coordenadas Geográficas del distrito son Latitud: $12^{\circ} 37^{\prime} 3^{\prime \prime}$ S y Longitud: $76^{\circ} 38^{\prime} 29^{\prime}$ ' W, y está ubicada a una altitud de 86.212 m.s.n.m

Los límites del Distrito son:

Por el Norte: $\quad$ Distrito de Chilca.

Por el Sur: Río Mala.

Por el Oeste: $\quad$ Distrito de San Antonio.

Por el Este: $\quad$ Distrito de Calango.

\section{MATERIALES}

Para lograr el desarrollo de la presente investigación fue importante hacer consultas bibliográficas y tesis sobre turismo, contar con carta del distrito a escala 1:50000, imágenes de satélites de global maper, fichas de encuestas, programas como ARGIS versión 10.1, AutoCAD versión 2017, cámaras fotográficas, GPS diferencial, Además de las entrevistas profundas a los administradores de hoteles, hospedajes y restaurantes ubicados en el área de estudio. Posterior a ello procesar la información a través de tablas y gráficos, interpretación, análisis, discusión y la conclusión para una posterior recomendación para el desarrollo económico del distrito a través del turismo.

\section{METODOLOGIA DE TRABAJO}

\section{Gabinete}

Se procedió a buscar información relativa sobre el tema de estudio y referente al área de estudio, luego de seleccionar y depurar la información que no era pertinente. Se procedió a realizar los cálculos del tamaño de la muestra para confeccionar la ficha de encuesta a la población, para poder planificar el trabajo de campo.

\section{Campo}

Se realizó en el año 2018 las encuestas a la población con apoyo de estudiantes de ingeniería geográfica del curso de catastro, distribuidos en Nuevo San Andrés, San Vicente de Azpitia y el centro poblado de santa cruz de flores. Por otro lado, el responsable del articulo realizo las encuestas a los restaurantes, hospedajes y hoteles en el distrito, también realice la entrevista a los sistemas de transporte local del distrito y las empresas de 
transportes de Lima a Mala

\section{Gabinete:}

Es el momento de procesar la información, obtener las tablas y gráficos para su posterior interpretación, discusión y análisis de la información, conclusiones y recomendaciones.

\section{RESULTADOS Y DISCUSIONES}

\section{Respecto del área del Servicio de Transporte para el turismo}

De la discusión de los valores de las tablas respecto de los tres elementos como son el transporte, hospedajes y restaurantes evaluados se puede interpretar lo siguiente:

Se puede colegir que, de la primera tabla, que la capacidad de transporte de ambas empresas tiene la capacidad para transportar 13924 personas por día desde Lima a San Pedro de Mala y viceversa.

Tabla 1. Capacidad de transporte total diario por las empresas desde Lima

\begin{tabular}{lccc}
\hline \multicolumn{1}{c}{ Nombre empresa } & $\mathrm{N}^{\circ}$ de unidades & Capacidad por viaje & Capacidad parcial dia \\
\hline E.T.COMPERSA 73 & 50 & 2551 & 10240 \\
E.T.SAN PEDRO & 17 & 921 & 3684 \\
\hline Capacidad total & 67 & 3472 & 13924 \\
\hline
\end{tabular}

De la Tabla y Figura 1 se aprecia que el total de buses de ambas empresas son 67, y pueden trasladar a 13924 personas por día desde Lima a San Pedro de Mala.

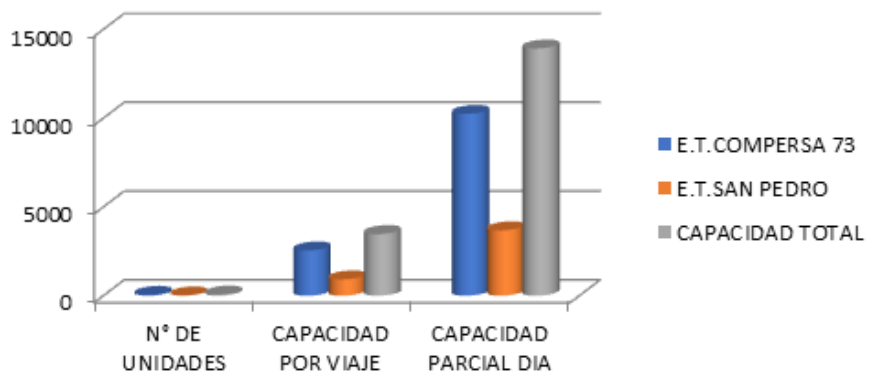

Figura 1. Capacidad de Transporte Total diario por las Empresas desde Lima. Fuente: Datos del Investigador (2017). Elaboración Propia (2019)

De la Tabla y Figura 2 se puede precisar que la capacidad de transporte local de ambas empresas es de 2880 personas por día de Santa Cruz de Flores a San Pedro de Mala a Santa Cruz de Flores.

Tabla 2. Capacidad de transporte total diario por las empresas desde Lima

\begin{tabular}{cccccc}
\hline Empresas & $\mathbf{N}^{\mathbf{o}}$ de Unidades & $\begin{array}{c}\text { Capacidad por } \\
\text { Unidad }\end{array}$ & $\begin{array}{c}\text { Capacidad } \\
\text { Total }\end{array}$ & Recorrido/día & Sub Total \\
\hline ETVC & 20 & 18 & 360 & 04 & 1,440 \\
ETSCM & 20 & 18 & 360 & 04 & 1,440 \\
\hline \multicolumn{7}{c}{ Capacidad Total de Transporte por día de las Empresas Locales } \\
\hline
\end{tabular}




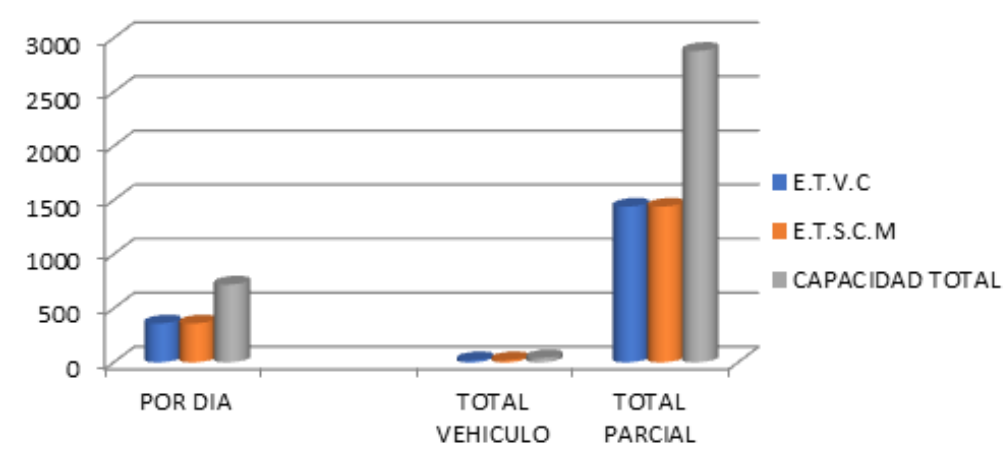

Figura 2. Capacidad de Transporte Diario por las dos Empresas. Fuente: Datos del Investigador (2017). Elaboración Propia (2019)

\section{Respecto del servicio de Hoteles y Hospedajes}

De la Tabla y Figura 3 se puede establecer que el total de hoteles y hospedajes del distrito de Santa Cruz de Flores son 12 y tiene una capacidad para 414 personas por día.

Tabla 3. Capacidad total hospedaje en el distrito Santa Cruz de Flores

\begin{tabular}{lccc}
\hline Lugar & Hospedaje & $\mathrm{N}^{\circ}$ de habitaciones & $\mathrm{N}^{\circ}$ de camas \\
\hline Santa Cruz de Flores & 6 & 58 & 151 \\
San Vicente de Azpitia & 6 & 70 & 263 \\
\hline Total & 12 & 128 & 414 \\
\hline
\end{tabular}

De la Tabla 3 se puede afirmar que la cantidad de camas es de 414 para atender a los turistas que visitan el distrito de Santa Cruz de Flores.

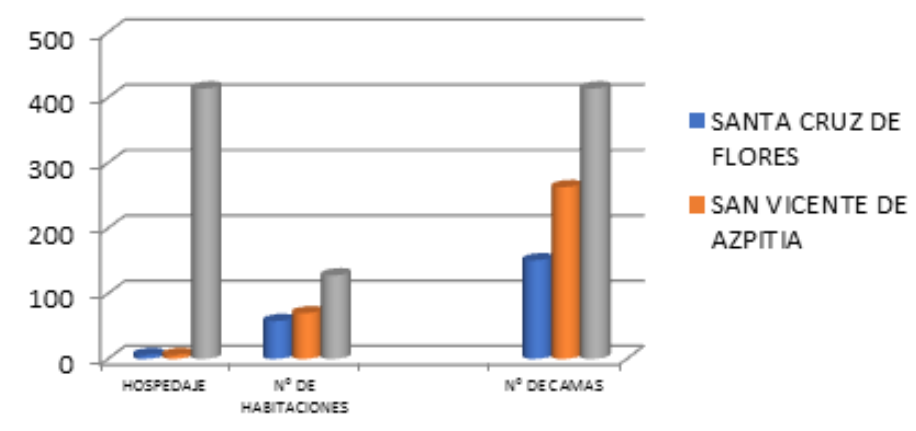

Figura 3. Capacidad total hotelera en el distrito Santa Cruz de Flores. Fuente: Datos del Investigador (2017). Elaboración Propia (2019)

\section{Respecto del servicio de Restaurantes}

De la Tabla y Figura 4 respecto a los restaurantes se puede indicar que hay 25 en el distrito con una capacidad de atención de 1289 personas por día.

Tabla 4. Capacidad total de restaurante en el distrito Santa Cruz de Flores

\begin{tabular}{lcccc}
\hline Ubicación de restaurantes & Cantidad & Mesas & Sillas & Capacidad parcial \\
\hline Santa Cruz de Flores & 17 & 145 & 627 & 627 \\
San Vicente de Azpitia & 8 & 135 & 662 & 662 \\
total & 25 & 280 & 1289 & 1289 \\
\hline
\end{tabular}


De la Tabla $N^{\circ} 04$ se puede afirmar que en el Distrito de Santa Cruz de Flores hay 25 restaurante con una capacidad de atención por día de 1289 turistas.

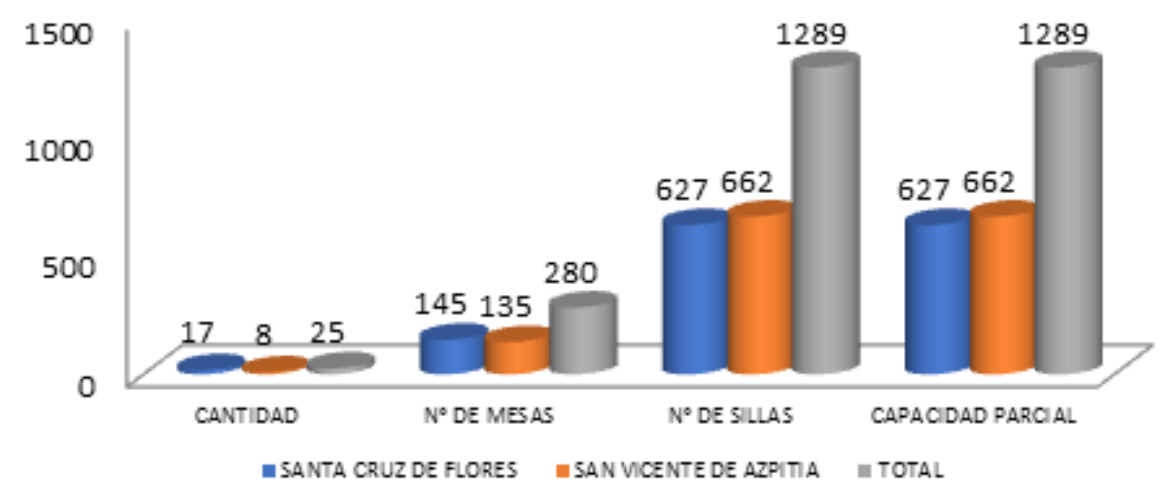

Figura 4. Capacudad total de restaurante del distrito Santa Cruz de Flores. Fuente: Datos del Investigador (2017). Elaboración Propia (2019).

Se ha tenido como resultado de las 4 tablas, que la capacidad de carga actual del flujo turístico en el distrito tiene un déficit de 386 camas para la atención al turista que llega al distrito, mientras que en el servicio de alimentación si hay capacidad para atender la demanda.

\section{CONCLUSIONES}

Es pertinente indicar que hay una demanda insatisfecha del turista en hospedaje.

No existe agencias de viaje ni guías de turismo.

Falta de mapas de las rutas de ciclismo de aventura.

Falta de señaléticas de todos los circuitos turísticos.

\section{Agradecimientos}

En primer lugar, agradecer a Dios por darme la vida, a mis padres: Antonio Arias Huapaya por confiar en mi capacidad y sabiduría, y del mismo modo a mi señora madre Dona Espichan Avalos, por sus sacrificios y sabios consejos.

A mi esposa Maruja por su apoyo y comprensión para darme aliento en los momentos más difíciles de la investigación, y a los estudiantes de ingeniería geográfica en especial del curso de catastro.

\section{Referencias}

Acerenza, Miguel Ángel. (2006). Política Turística y Planificación del Turismo. México- D.F.: Trillas S.A. de C.V.

Aguilar, Víctor. Leonith Hinojosa y Carlos Milla. (1992). TURISMO Y DESARROLLO. CUSCOPERU: CBS-Bartolomé de las Casas.

Achahui Loaiza, Luis (2004). Nueva Ley Orgánica de Municipalidades-Ley N²7972. Lima-Perú: 


\section{EDIGRABER.}

Andrés Ziperovich. (2010). Turismo y Recreación. México D.F.: Trillas, S.A. de C.V.7.

Aranda, D., Palomino, R., Ricketts, E., y Tapia, C. (2006). Planeamiento Estratégico para el Desarrollo de la Macroregión Sur del Perú. Tesis para obtener el Grado de Magíster en Administración Estratégica de Empresas. Centro de Negocios de la Pontificia Universidad Católica del Perú, Lima, Perú.

Caballero Romero, Alejandro. (2012). Metodología integral innovadora para planes y tesis. La Molina- Lima 12, Perú: Instituto Metodológico Alen Caro E.I.R.L.

Cabrera Sandoval, Patricia y Sullca Lorenzo, Susana, 2015. Tesis: "El Turismo una Alternativa para el Desarrollo del Distrito de Santa Rosa de Ocopa-2015”.

Cruz Ledesma, Sigrid Stefani (2006). Turismo Alternativa de Desarrollo de los Pueblos y Regiones del Perú. Caso: Distrito Callahuanca, Provincia de Huarochirí, Departamento de Lima. Tesis para obtener el título en Administración de Empresas y Negocios Internacionales. Universidad Nacional Mayor de San Marcos, Lima, Perú.

Cruz Reyes, Fray Masías (2006). Ordenamiento Territorial instrumento de Desarrollo del turismo sostenible. Caso: Cuenca del rio negro, Provincia de Huaraz, Departamento de Ancash. Tesis para obtener el grado de magister en Geografía. Universidad Nacional Mayor de San Marcos, Lima, Perú.

De la Torre, Francisco. (1996). Introducción al Estudio del Turismo. México-D.F.: Editorial Continental, S.A. de C.V.

Figueroa Pinedo, Jessica Ruth, 2013. Tesis: "TURISMO, POBREZA Y DESARROLLO SOSTENIBLE EN EL PERU: LOS CASOS DE CUZCO, CAJAMARCA Y LA LIBERTAD”.

García, F., Hanco, H., Marca, M., y Zea, R. (2009). Plan Estratégico para el Desarrollo Turístico Ccatcca - Ocongate Cusco. Tesis para obtener el Grado de Magíster en Administración Estratégica de Empresas. Centro de Negocios de la Pontificia Universidad Católica del Perú, Lima, Perú.

Hernández Sampieri Roberto, Carlos Fernández-Collado y Pilar Baptista Lucio. (2006). Metodología de la investigación. Iztapalapa - México: McGraw-Hill Interamericana.

Hernández Sampieri Roberto, Carlos Fernández Collado y Pilar Baptista Lucio. (2014). Metodología de la Investigación. México: McGraw-Hill-Interamericana Editores S.A. DE C.V.

Hollman Sepúlveda, Rafael Basurto, Yuri Vizcarra. (2010) Plan Estratégico para el Desarrollo del Turismo Rural Comunitario en la Región Cusco. Tesis para obtener el título en Administración Estratégica de Empresas. Centro de Negocios de la Pontificia Universidad Católica del Perú, Lima, Perú. 